\title{
MARTIN COMPACTIFICATIONS OF THE PUNCTURED DISK WITH CLOSE TO ROTATION FREE DENSITIES
}

\author{
TOSHIMASA TADA
}

(Communicated by Irwin Kra)

\begin{abstract}
The purpose of this paper is to prove that Martin compactifications $\Omega_{P}^{*}$ and $\Omega_{Q}^{*}$ of the punctured unit disk $\Omega: 0<|z|<1$ with respect to equations $\Delta u=P u$ and $\Delta u=Q u$, respectively, are homeomorphic to each other if $|P(z)-Q(z)|=O\left(|z|^{-2}\right)(z \rightarrow 0)$ and $P(z)=P(|z|)(z \in \Omega)$.
\end{abstract}

Before stating our result more precisely we start fixing terminologies. We denote by $\Omega$ the punctured unit disk $0<|z|<1$ which is viewed as an end of the punctured sphere $0<|z| \leq \infty$ so that the unit circle $|z|=1$ is the relative boundary $\partial \Omega$ of $\Omega$ and the origin $z=0$ is the ideal boundary $\delta \Omega$ of $\Omega$. By a density $P$ on $\Omega$ we mean a nonnegative locally Hölder continuous function $P(z)$ on $\bar{\Omega}=\Omega \cup \partial \Omega$. For a density $P$ on $\Omega$ we consider the Martin compactification $\Omega_{P}^{*}$ of $\Omega$ with respect to the equation

$$
L_{P} u \equiv \Delta u-P u=0 \quad\left(\Delta=\partial^{2} / \partial x^{2}+\partial^{2} / \partial y^{2}\right)
$$

on $\Omega([3]$, cf. also $[6,1])$. In the case that $P$ is a rotation free density on $\Omega$, i.e. a density $P$ on $\Omega$ satisfying $P(z)=P(|z|)(z \in \Omega)$, the Martin compactification $\Omega_{P}^{*}$ is characterized completely by Nakai [4] in terms of a singularity index $\alpha(P)$ of $P$ at $\delta \Omega$ :

$$
\Omega_{P}^{*} \cong\{\alpha(P) \leq|z| \leq 1\},
$$

i.e. the homeomorphism $\pi_{P}$ from $\Omega$ to $\alpha(P)<|z|<1$ defined by

$$
\pi_{P}(z)=(\alpha(P)+(1-\alpha(P))|z|) z /|z|
$$

can be extended to a homeomorphism $\pi_{P}^{*}$ from $\Omega_{P}^{*}$ to $\alpha(P) \leq|z| \leq 1$ and every point in the ideal boundary $\Omega_{P}^{*}-\Omega$ of $\Omega$ is minimal. Here $\alpha(P)$ is a value in $[0,1)$ depending on the singular behavior of $P$ at $\delta \Omega(\mathrm{cf} . \S 1)$ and the only relevant fact about $\alpha(P)$ for the Martin compactification is whether $\alpha(P)=0$ or not. For example, $\alpha(P)$ of the density $P(z)=|z|^{3}$ on $\Omega$ satisfies $[\mathbf{4}, 5]$

$$
\alpha(P)=0 \text { if and only if }-2 \leq s \leq \infty,
$$

and hence

$$
\Omega_{P}^{*} \cong\{|z| \leq 1\} \text { if }-2 \leq s \leq \infty
$$

This fact is partly extended to $\Omega_{P}^{*}$ with a general density $P$ on $\Omega$ by Kawamura [2]: a general density $P$ on $\Omega$ satisfies

$$
\Omega_{P}^{*} \cong\{|z| \leq 1\} \quad \text { if } P(z)=O\left(|z|^{-2}\right) \quad \text { as } z \rightarrow 0 .
$$

Received by the editors November 4, 1986 and, in revised form, February 24, 1987.

1980 Mathematics Subject Classification (1985 Revision). Primary 31A35; Secondary 30F25.

The author was supported in part by Grant-in-Aid for Scientific Research, No. 60302004, Japanese Ministry of Education, Science and Culture. 
In this paper we show that $\Omega_{Q}^{*}$ with a general density $Q$ on $\Omega$ is homeomorphic to $\Omega_{P}^{*}$ with a rotation free density $P$ on $\Omega$ if $Q$ is close to $P$.

THEOREM. Let $P$ be a rotation free density on $\Omega$ and $Q$ be a general density on $\Omega$ with

$$
|P(z)-Q(z)|=O\left(|z|^{-2}\right) \quad(z \rightarrow 0),
$$

then $\Omega_{P}^{*} \cong \Omega_{Q}^{*}$, i.e. the identity mapping $I_{\Omega}$ of $\Omega$ is extended to a homeomorphism $I_{\Omega}^{*}$ from $\Omega_{P}^{*}$ to $\Omega_{Q}^{*}$ and every point in $\Omega_{Q}^{*}-\Omega$ is minimal.

From this theorem (1) follows since $\alpha(P)=0$ if $P \equiv 0$.

The author is very grateful to Professor M. Nakai for his helpful suggestions.

1. Singularity indices. We recall the definition and fundamental properties of singularity indices of rotation free densities on $\Omega$ according to [4].

Let $P$ be a rotation free density on $\Omega$. The unique bounded solution $e_{P}$ of $L_{P} u=0$ on $\Omega$ with boundary values 1 on $\partial \Omega$ is referred to as the $P$-unit on $\Omega$. We also consider rotation free densities $P_{n}(n=0,1, \ldots)$ on $\Omega$ defined by $P_{n}(z)=P(z)+n^{2}|z|^{-2}(z \in \Omega)$ and denote by $e_{n}$ the $P_{n}$-unit on $\Omega$, where we follow the convention $P_{0}=P$ and $e_{0}=e_{P}$. Then $e_{n}$ is positive on $\Omega$ and rotation free, i.e. $e_{n}(z)=e_{n}(|z|)(z \in \Omega)$. Each function $e_{n}(r) / e_{0}(r)(n=1,2, \ldots)$ of $r$ in $(0,1]$ is increasing so that there exists the limit

$$
\alpha_{n}(P)=\lim _{r \rightarrow 0} \frac{e_{n}(r)}{e_{0}(r)}
$$

which is referred to as the $n$th singularity index of $P$ at $\delta \Omega$. In particular we denote by $\alpha(P)$ the 1st singularity index $\alpha_{1}(P)$ and call it simply the singularity index of $P$ at $\delta \Omega$. Then we have the following fundamental inequalities [4]

$$
0 \leq \alpha(P)^{\left(3^{n}-1\right) / 2} \leq \alpha_{n}(P) \leq \alpha(P)^{n}<1 \quad(n=1,2, \ldots) .
$$

2. Comparison between Martin compactifications. For general densities $P$ and $Q$ on $\Omega$ with $P \leq Q$, we show that $\Omega_{P}^{*} \cong \Omega_{Q}^{*}$ if the $Q$-Green's function is comparable with the $P$-Green's function.

Consider general densities $P$ and $Q$ on $\Omega$ with $P(z) \leq Q(z)(z \in \Omega)$. With the density $P(Q$, resp.) we associate the class $P P(\Omega ; \partial \Omega)(Q P(\Omega ; \partial \Omega)$, resp.) of nonnegative solutions $u$ of $L_{P} u=0\left(L_{Q} u=0\right.$, resp.) on $\Omega$ with vanishing boundary values on $\partial \Omega$. For any function $u$ in $P P(\Omega ; \partial \Omega)$, the sequence $\left\{v_{n}\right\}_{2}^{\infty}$ of solutions $v_{n}$ of $L_{Q} v=0$ on $\{1 / n<|z|<1\}$ with boundary values $u$ on $\{|z|=1 / n\} \cup\{|z|=1\}$ is decreasing. Then $\left\{v_{n}\right\}$ converges to a function $Q_{u}$ in $Q P(\Omega ; \partial \Omega)$ uniformly on every compact subset of $\Omega$. Similarly we may construct a function $P_{v}$ in $P P(\Omega ; \partial \Omega) \cup\{\infty\}$ for every $v$ in $Q P(\Omega ; \partial \Omega)$. We remark that $Q_{u} \leq u, P_{v} \geq v, \lambda Q_{u} \not u$, and $P_{v} \geq \lambda v$ for any constant $\lambda$ with $\lambda>1$ if $Q_{u} \neq 0$ and $P_{v} \neq \infty$. Assume that $u$ is a minimal function in $P P(\Omega ; \partial \Omega)$ and $v$ is a function in $Q P(\Omega ; \partial \Omega)$ with $u \geq v>0$. Set $\lambda_{0}=\max \left\{\lambda: Q_{u} \geq \lambda v\right\}$. Then $\lambda_{0} \geq 1$ by $Q_{u} \geq v$. Further $u \geq \lambda_{0} v$ implies $u \geq P_{\lambda_{0} v} \geq \lambda_{0} v$, and hence $P_{\lambda_{0} v}=\mu u$ for a constant $\mu$ with $0<\mu \leq 1$. Therefore $u=P_{\left(\lambda_{0} / \mu\right) v} \geq\left(\lambda_{0} / \mu\right) v$ implies $Q_{u} \geq\left(\lambda_{0} / \mu\right) v$ so that $\mu=1$ by the definition of $\lambda_{0}$. Thus $u \geq P_{Q_{u}}=P_{\lambda_{0} v}+P_{Q_{u}-\lambda_{0} v}=u+P_{Q_{u}-\lambda_{0} v}$ and $0 \leq Q_{u}-\lambda_{0} v \leq P_{Q_{u}-\lambda_{0} v}$ yield $Q_{u}=\lambda_{0} v$. Then we have the following lemma. 
LEMMA 1. Let $u$ be a minimal function in $P P(\Omega ; \partial \Omega)$. Then

$$
\{v \in Q P(\Omega ; \partial \Omega): u \geq v\}=\left\{\lambda Q_{u}: 0 \leq \lambda \leq 1\right\},
$$

and hence $Q_{u}$ is minimal if $Q_{u} \neq 0$.

We have also the following

LEMMA 2. Let $u_{1}$ and $u_{2}$ be minimal functions in $P P(\Omega ; \partial \Omega)$. Then $\{v \in$ $\left.Q P(\Omega ; \partial \Omega): u_{1} \geq v, u_{2} \geq v\right\}=\{0\}$ if $u_{1}$ is nonproportional to $u_{2}$.

In fact, if a function $v$ in $Q P(\Omega ; \partial \Omega)-\{0\}$ is dominated by $u_{1}$ and $u_{2}$, then $P_{v}$ is proportional to $u_{1}$ and $u_{2}$.

We denote by $G_{P}\left(G_{Q}\right.$, resp.) the $P$-Green's ( $Q$-Green's, resp.) function on $\Omega$, i.e. the Green's function with respect to $L_{P} u=0\left(L_{Q} u=0\right.$, resp.). Since $P$ is dominated by $Q, G_{P}$ dominates $G_{Q}$. Assume that there exist a point $z_{0}$ in $\Omega$, a positive constant $C$, and a positive number $\delta$ with $\delta<1$ such that

$$
C G_{P}\left(z_{0}, \varsigma\right) \leq G_{Q}\left(z_{0}, \varsigma\right) \quad(0<|\varsigma|<\delta) .
$$

Then the $P$-Martin kernel $K_{P}(z, \varsigma)=G_{P}(z, \varsigma) / G_{P}\left(z_{0}, \varsigma\right)$ on $\Omega$ and the $Q$-Martin kernel $K_{Q}(z, \varsigma)=G_{Q}(z, \varsigma) / G_{Q}\left(z_{0}, \varsigma\right)$ on $\Omega$ satisfy

$$
K_{P}(z, \zeta) \geq C K_{Q}(z, \varsigma) \quad(z \in \Omega, 0<|\zeta|<\delta) .
$$

Let $\Gamma_{P}^{*}\left(\Gamma_{Q}^{*}\right.$, resp.) be the set of points in $\Omega_{P}^{*}-\Omega\left(\Omega_{Q}^{*}-\Omega\right.$, resp.) over $\delta \Omega$ and $\varsigma^{*}$ be any point in $\Gamma_{P}^{*}$. Take a sequence $\left\{\zeta_{n}\right\}_{1}^{\infty}$ of points $\zeta_{n}$ in $\Omega$ with $\lim \zeta_{n}=\varsigma^{*}$ in $\Omega_{P}^{*}$. Since $\lim \left|\zeta_{n}\right|=0$, a subsequence of $\left\{\zeta_{n}\right\}$ converges to a point $\xi^{*}$ in $\Gamma_{Q}^{*}$. In view of (4) we have $K_{P}\left(z, \varsigma^{*}\right) \geq C K_{Q}\left(z, \xi^{*}\right)$ so that by Lemma $1 \xi^{*}$ is independent of the choice of the sequence $\left\{\zeta_{n}\right\}$ and its subsequence when $\zeta^{*}$ is minimal. Now assume that every point in $\Gamma_{P}^{*}$ is minimal. Then we may extend the identity mapping $I_{\Omega}$ of $\Omega$ to a mapping $I_{\Omega}^{*}$ from $\Omega_{P}^{*}$ to $\Omega_{Q}^{*}$. We remark that every point in $\Omega_{P}^{*}-\left(\Omega \cup \Gamma_{P}^{*}\right)\left(\Omega_{Q}^{*}-\left(\Omega \cup \Gamma_{Q}^{*}\right)\right.$, resp. $)$ is minimal and a neighborhood of $\Omega_{P}^{*}-\left(\Omega \cup \Gamma_{P}^{*}\right)$ $\left(\Omega_{Q}^{*}-\left(\Omega \cup \Gamma_{Q}^{*}\right)\right.$, resp. $)$ is homeomorphic to a neighborhood of $\{|z|=1\}$ in $\{|z| \leq 1\}$ since $P(Q$, resp.) is Hölder continuous on $\bar{\Omega}$ (cf. e.g. $[\mathbf{1}, \mathbf{4}])$. Clearly $I_{\Omega}^{*}$ is surjective. Further $I_{\Omega}^{*}$ is injective by Lemma 2 . Thus we deduce the following

LEMMA 3. If any point in $\Gamma_{P}^{*}$ is minimal and if (3) is valid, then $\Omega_{P}^{*} \cong \Omega_{Q}^{*}$, i.e. $I_{\Omega}$ can be extended to a homeomorphism from $\Omega_{P}^{*}$ to $\Omega_{Q}^{*}$ and any point in $\Gamma_{Q}^{*}$ is minimal.

3. Proof of the theorem. Let $P$ be a rotation free density on $\Omega$ and $Q$ be a general density on $\Omega$ with $|P(z)-Q(z)|=O\left(|z|^{-2}\right)(z \rightarrow 0)$. There exists a positive integer $k$ such that the rotation free density $R$ on $\Omega$ given by $R(z)=$ $\max \left(P(z)-\dot{\kappa}^{2}|z|^{-2}, 0\right)(z \in \Omega)$ satisfies

$$
\begin{gathered}
R(z) \leq P(z) \leq R(z)+k^{2}|z|^{-2} \\
R(z) \leq Q(z) \leq R(z)+(2 k)^{2}|z|^{-2} .
\end{gathered}
$$

We assume that $\alpha(R)>0$ since we have seen [7] that $\alpha(R)=0$ implies $\Omega_{P}^{*} \cong$ $\{|z| \leq 1\}$ and $\Omega_{Q}^{*} \cong\{|z| \leq 1\}$. We denote by $f_{n}(n=0,1, \ldots)$ then $R_{n}$-unit and set

$$
\begin{aligned}
M_{R} & =\max \left\{G_{R}\left(\frac{1}{2}, \varsigma\right):|\varsigma|=1 / 4\right\}, \\
m_{P} & =\min \left\{G_{P}\left(\frac{1}{2}, \varsigma\right):|\varsigma|=1 / 4\right\} .
\end{aligned}
$$


Then we have

$$
\begin{array}{ll}
G_{R}\left(\frac{1}{2}, \varsigma\right) / M_{R} \leq f_{0}(\varsigma) / f_{0}\left(\frac{1}{4}\right) & (0<|\varsigma|<1 / 4), \\
G_{P}\left(\frac{1}{2}, \varsigma\right) / m_{P} \geq f_{k}(\varsigma) / f_{k}\left(\frac{1}{4}\right) & (0<|\varsigma|<1 / 4) .
\end{array}
$$

Further we have $\alpha_{k}(R) f_{0}(\varsigma) \leq f_{k}(\varsigma)(\varsigma \in \Omega)$ and $\alpha_{k}(R)>0$ by (2). Therefore the positive constant

$$
C_{P}=m_{P} f_{0}\left(\frac{1}{4}\right) \alpha_{k}(R) / M_{R} f_{k}\left(\frac{1}{4}\right)
$$

satisfies

$$
C_{P} G_{R}\left(\frac{1}{2}, \varsigma\right) \leq G_{P}\left(\frac{1}{2}, \varsigma\right) \quad(0<|\varsigma|<1 / 4)
$$

Similarly an inequality

$$
C_{Q} G_{R}\left(\frac{1}{2}, \varsigma\right) \leq G_{Q}\left(\frac{1}{2}, \varsigma\right) \quad(0<|\varsigma|<1 / 4)
$$

is valid for a positive constant $C_{Q}$. Thus Lemma 3 and the fact that any point in $\Omega_{R}^{*}-\Omega$ is minimal yield that $\Omega_{P}^{*} \cong \Omega_{R}^{*}, \Omega_{Q}^{*} \cong \Omega_{R}^{*}$, and hence the theorem is proved.

\section{REFERENCES}

1. S. Itô, Martin boundary for linear elliptic differential operator of second order in a manifold, $\mathrm{J}$. Math. Soc. Japan 16 (1964), 307-334.

2. M. Kawamura, On a conjecture of Nakai on Picard principle, J. Math. Soc. Japan 31 (1979), $359-371$.

3. M. Nakai, The space of non-negative solutions of the equation $\Delta u=p u$ on a Riemann surface, Kōdai Math. Sem. Rep. 12 (1960), 151-178.

4. $\ldots$, Martin boundary over an isolated singularity of rotation free density, J. Math. Soc. Japan 26 (1974), 483-507.

5. 412-431.

6. Sur, The Martin boundary for a linear elliptic second order operator, Izv. Akad. Nauk USSR 27 (1963), 45-60 (Russian).

7. T. Tada, The role of boundary Harnack principle in the study of Picard principle, J. Math. Soc. Japan 34 (1982), 445-453.

Department of Electrical and CoMputer EngineERing, Nagoya Institute OF TEChNOLOGY, GoKiso Showa, NAgoya 466, JAPAN

Current address: Department of Mathematics, Daido Institute of Technology, Daido, Minami, Nagoya 457, Japan 